Ciência Florestal, Santa Maria, v. 22, n. 3, p. 579-588, jul.-set., 2012

ISSN 0103-9954

\title{
CARACTERÍSTICAS FÍSICO-QUÍMICAS E ENERGÉTICAS DE DUAS ESPÉCIES DE OCORRÊNCIA NO SEMIÁRIDO BRASILEIRO
}

\author{
PHYSICOCHEMICAL AND ENERGETIC CHARACTERISTICS OF TWO \\ SPECIES OCCURRING IN THE BRAZILIAN SEMIARID
}

\author{
Pedro Nicó de Medeiros Neto ${ }^{1}$ Elisabeth de Oliveira ${ }^{2}$ Leandro Calegari ${ }^{3}$ \\ Antonio Marcos César de Almeida ${ }^{4}$ Alexandre Santos Pimenta ${ }^{5}$ Angélica de Cássia Oliveira Carneiro ${ }^{6}$
}

\section{RESUMO}

A vegetação nativa da Região Nordeste vem sendo explorada e utilizada de forma intensiva e de maneira inadequada para finalidades energéticas e nas construções rurais, tornando-se evidente a importância dos conhecimentos relativos às características da madeira. Assim, essa pesquisa teve como objetivo avaliar as características físico-químicas e energéticas da madeira, os rendimentos da carbonização e a caracterização do carvão vegetal das espécies Poincianella pyramidalis Tul. L. P. Queiroz (Catingueira) e Handroanthus impertiginosus (Mart. ex DC.) Mattos (Pau-d'arco). Para atender ao objetivo, foram abatidas cinco árvores de cada espécie, amostradas aleatoriamente na Fazenda Santa Bárbara, localizada no município de São Mamede-PB. As árvores foram devidamente identificadas e transportadas para o Setor de Tecnologia de Produtos Florestais (STPF) da Universidade Federal de Campina Grande. De cada árvore, foram retirados toretes de $30 \mathrm{~cm}$ de altura a 0 (base), 25, 50, 75 e $100 \%$ da altura comercial do tronco, considerada até $5 \mathrm{~cm}$ de diâmetro. Da porção mediana de cada torete, foi retirado um disco de $2,5 \mathrm{~cm}$ de espessura, subdividido em quatro partes, em forma de cunha, passando pela medula. Duas cunhas opostas foram utilizadas para a determinação da densidade básica, e as restantes foram reservadas para a realização das análises físicas, químicas e energéticas da madeira, os rendimentos em carbonização e a caracterização do carvão produzido pelas espécies estudadas. A densidade básica foi determinada de acordo com o método da balança hidrostática e para a determinação da densidade básica média de cada árvore utilizou-se como fator de ponderação o volume entre seções de cada disco. Após a secagem ao ar, as amostras destinadas às análises químicas foram transformadas em serragem e feitas determinações quantitativas de extrativos totais, da lignina, das cinzas, e o teor de holocelulose foi estimado por diferença. As amostras destinadas à carbonização foram transformadas em cavacos, e carbonizadas em forno elétrico (mufla) por cinco horas e meia. Foram feitas determinações dos rendimentos dos produtos das carbonizações, e das propriedades físicas e químicas do carvão vegetal. De modo geral, as madeiras estudadas apresentaram características físicas semelhantes. Com relação às características químicas, o pau-d'arco apresentou maior teor de lignina $(28,40 \%)$, e não houve diferença significativa entre as espécies para o teor de holocelulose. O poder calorífico superior da catingueira exibiu os menores valores, para a madeira $\left(4.413,50 \mathrm{kcal}^{\mathrm{kg}} \mathrm{kg}^{-1}\right)$ e carvão vegetal $\left(6.247,80 \mathrm{kcal} . \mathrm{kg}^{-1}\right)$, respectivamente. A catingueira apresentou um maior rendimento em carvão $(43,03 \%)$, mas de qualidade inferior. O carvão vegetal do pau-d'arco apresentou o maior teor de carbono

1. Engenheiro Florestal, Mestrando do Programa de Pós-graduação em Ciências Florestais, Unidade Acadêmica de Engenharia Florestal, Universidade Federal de Campina Grande, Caixa Postal 064, CEP 58700-970, Patos (PB). pedroflorestal@gmail.com

2. Engenheira florestal, Dr., Professora Adjunta da Unidade Acadêmica de Engenharia Florestal, Universidade Federal de Campina Grande, Caixa Postal 064, CEP 58700-970, Patos (PB). betholiveira@gmail.com

3. Engenheiro Florestal, Dr., Professor Adjunto da Unidade Acadêmica de Engenharia Florestal, Universidade Federal de Campina Grande, Caixa Postal 064, CEP 58700-970, Patos (PB). leandrocalegari@yahoo.com.br

4. Engenheiro Florestal, Mestrando do Programa de Pós-graduação em Ciências Florestais, Unidade Acadêmica de Engenharia Florestal, Universidade Federal de Campina Grande, Caixa Postal 064, CEP 58700-970, Patos (PB). marcosengflorestal@hotmail.com

5. Engenheiro Florestal, Dr., Professor Adjunto do Departamento de Engenharia Florestal, Universidade Federal do Rio Grande do Norte, CEP 59.072-970, Lagoa Nova (RN). aspimenta@ufrnet.br

6. Engenheira Florestal, Dr., Professora Adjunta do Departamento de Engenharia Florestal, Universidade Federal de Viçosa, CEP 36570-000, Viçosa (MG). cassiacarneiro1@gmail.com

Recebido para publicação em 15/04/2011 e aceito em 12/07/2011

Ci. Fl., v. 22, n. 3, jul.-set., 2012 
fixo $(67,68 \%)$. As espécies apresentaram densidade verdadeira do carvão e rendimento em carbono fixo semelhantes. Pode-se concluir que as duas espécies de ocorrência no semiárido Nordestino, apresentam boas características para produção de carvão. De modo geral, o pau-d'arco apresentou as melhores propriedades para fins energéticos.

Palavras-chave: Caatinga; madeira; carvão vegetal.

\begin{abstract}
The native vegetation from Brazilian Northeast has been explored and used intensively and inadequately as a source of energy and material for rural constructions. Now, it is recognized how important it is to get to know the characteristic of the wood produced by this vegetation. Thus, this study aims to provide information on the physicochemical and energetic characteristics of wood, carbonization yields and characterization of the charcoal from wood of Poincianella pyramidalis Tul. L.P.Queiroz and Handroanthus impertiginosus (Mart. ex DC.) Mattos. To meet the goal five trees from each species were cut down and randomly sampled at Santa Barbara Farm, located in the municipality of São Mamede-PB. The trees were properly identified and transported to the Department of Forest Products Technology (STPF) at the Federal University of Campina Grande. Thirty centimeters sections were removed from each tree at 0 (base), 25, 50, 75 and $100 \%$ of the commercial height (trunk diameter $>5 \mathrm{~cm}$ ). A $2.5 \mathrm{~cm}$ thick disc was removed from the median part of each $30 \mathrm{~cm}$ section, and each disc was subdivided in four wedge pieces, passing through the trunk pith. Two opposite wedge pieces were used to determine the basic density of wood, ant the other two pieces were put aside in order to carry out physical, chemical and energetic analyses, carbonization yields and characterization of the charcoal produced by the studied species. Basic density was determined according to the method of hydrostatic balance and to determine the average basic density of each tree, the volume between sections of each disc was used as a weighting factor. After the air drying, the samples intended for chemical analysis were transformed into sawdust and quantitative determinations of total extractives were performed, lignin, ashes, and the holocellulose content was estimated by difference. The samples intended for carbonization were processed into chips, and carbonized in an electric oven (furnace) for $5 \mathrm{~h} 30 \mathrm{mim}$. Determinations of the yield of products of carbonization were carried out as well as physical and chemical properties of wood charcoal. In general, the studied wood showed similar physical characteristics. Regarding to chemical properties, Pau-d'arco wood showed a higher lignin content (28.40\%), and there were not any significant differences among species for the holocellulose content The superior calorific power of Catingueira wood $\left(4413.50 \mathrm{kcal} . \mathrm{kg}^{-1}\right)$ showed the lowest values for wood $\left(4413.50 \mathrm{kcal} . \mathrm{kg}^{-1}\right)$ and charcoal (6247.80 kcal. $\left.\mathrm{kg}^{-1}\right)$ respectively. The catingueira wood showed a higher charcoal yield (43.03\%), but lower quality. The Pau-d'arco charcoal had the highest fixed carbon content (67.68\%). These species showed similar charcoal true density and fixed carbon production. It is concluded that the two species which occurs in the Northeastern semiarid, have good properties for charcoal production. The Pau-d'arco showed the best properties for energy use.
\end{abstract}

Keywords: Caatinga; wood; charcoal.

\section{INTRODUÇÃO}

As novas restrições ecológicas ao uso de combustíveis fósseis e o aumento crescente do consumo energético têm realçado o interesse do uso da biomassa para produção de energia, em virtude de ser essa uma matéria-prima renovável e menos poluente quando comparada aos produtos não renováveis (PINHEIRO; SÉYE, 1998).

A Caatinga é o único bioma exclusivamente brasileiro, com uma área de $844.453 \mathrm{~km}^{2}$, apresentando uma área de cobertura vegetal nativa da ordem de $518.635 \mathrm{~km}^{2}$, o que equivale a $62,77 \%$ da área mapeada do bioma (MMA, 2007). O bioma representa aproximadamente $54 \%$ da região Nordeste e $11 \%$ do território brasileiro. Está compreendido entre os paralelos de $2^{\circ} 54^{\prime} \mathrm{S}$ a $17^{\circ} 21^{\prime} \mathrm{S}$ e envolve áreas dos estados do Ceará, Rio Grande do Norte, Paraíba, Pernambuco, Alagoas, Sergipe, sudoeste do Piauí, partes do interior da Bahia e do norte de Minas Gerais (ANDRADE et al., 2005). 
Araújo et al. (2007) relatam que a cobertura florestal da região Semiárida tem se reduzido drasticamente, fato ocasionado por falta de manejo adequado e pelo tipo de exploração adotado. Enfatizam que a exploração da vegetação da Caatinga é basicamente realizada com finalidade energética, principalmente no consumo da lenha e na produção de carvão vegetal.

A demanda de lenha anual, no estado da Paraíba, é de 3.983.634,23 st/ano, provocando um desmatamento de $42.524,77 \mathrm{ha} /$ ano de mata nativa. Desse total, aproximadamente $83 \%$ é utilizado no setor domiciliar, na forma de lenha e carvão vegetal (SUDEMA, 2004).

Johnson (1985) enfatiza que as espécies produtoras de carvão na Caatinga são: juremapreta (Mimosa tenuiflora (Will.) Poir.), catingueira (Poincianella pyramidalis (Tul.) L. P. Queiroz), angico (Anadenanthera colubrina (Vell.) Brenan. var. cebil (Gris.) Alts.), jucá (Caesalpinia ferrea Mart. ex Tul.), mororó (Bauhinia cheilantha (Bong) D. Dietr.), pereiro (Aspidospema pyrifolium Mart.) e craibeira (Tabebuia áurea (Silva Manso) S. Moore). O esgotamento das espécies madeireiras de uso tradicional da vegetação da Caatinga, consolidado com o aumento do uso da madeira em nível global, vem exigindo um maior conhecimento tecnológico sobre a utilização racional das essências florestais.

Apesar da importância da vegetação da caatinga como fonte de energia, ainda é grande a carência de informações, principalmente quanto ao aspecto tecnológico, ou seja, sobre as características de qualidade da madeira e do carvão produzido pelas espécies do semiárido (OLIVEIRA, 2003).

A catingueira (Poincianella pyramidalis Tul. L.P. Queiroz) pertence à família Fabaceae. É uma das espécies de maior ocorrência no semiárido nordestino. É encontrada nos estados do Piauí, Ceará, Rio Grande do Norte, Paraíba, Pernambuco, Alagoas, Sergipe e Bahia (MAIA, 2004; SIQUEIRA FILHO et al., 2009). Sua madeira é pesada, com densidade básica aproximada de 0,99 g. $\mathrm{cm}^{-3}$, contendo grande quantidade de celulose e lignina, sendo utilizada na construção civil, lenha, carvão, estacas, mourões, entre outros (MAIA, 2004; PAULA; ALVES, 2007).

O pau-d'arco (Handroanthus impertiginosus (Mart. ex DC.) Mattos) pertence à família Bignoniaceae. Ocorre, na América Latina, do México até a Argentina e, no Brasil, desde o Piauí e Ceará até Minas Gerais, Goiás e São Paulo
(LORENZI, 2002; MAIA, 2004; SABONARO; GALBIATTI, 2007; SIQUEIRA FILHO et al., 2009). A madeira é pesada, com densidade básica aproximada de $0,89 \mathrm{~g} . \mathrm{cm}^{-3}$, de excelente qualidade, resistente, empregada como pontes, dormentes, mourões, postes, caibros, construção civil e naval. Fornece lenha de boa qualidade (MAIA, 2004; PAULA; ALVES, 2007).

A descrição anatômica (BURGER; RICHTER, 1991), a composição química (SJÖSTRÖM, 1993) e a densidade (VITAL, 1984) formam os parâmetros que constituem a base para quaisquer estudos tecnológicos que sejam efetuados na madeira, auxiliando na interpretação dos resultados e permitindo empregá-la corretamente para determinado tipo de uso.

A densidade representa a quantidade de material lenhoso por unidade de volume ou, de forma inversa, o volume dos espaços vazios presentes na madeira, sendo um índice significativamente utilizado para avaliar a viabilidade do emprego da madeira para diversas finalidades (LIMA et al., 2000; VALÉRIO et al., 2008). É uma das propriedades que contribui com um maior número de informações sobre as suas características, devido a sua relação com sua resistência e rigidez (PANSHIN; DE ZEEUW, 1980; HAYGREEN; BOWYER, 1982 apud MELO et al., 2006; OLIVEIRA et al., 2007). No entanto, segundo Wenzl (1970), Brasil et al. (1977), apud Oliveira (2003), a densidade não deve ser isoladamente considerada um único índice de qualidade da madeira. A composição química e as características anatômicas também devem ser avaliadas.

Na produção de carvão vegetal, a densidade da madeira deve ser analisada sob vários aspectos, podendo várias considerações serem feitas em torno da mesma. Esta afeta a capacidade de produção de carvoaria, porque para um determinado volume de forno a utilização de madeira mais densa resulta em maior produção em peso. Além disto, madeira mais densa produz carvão com densidade mais elevada, com vantagens para alguns de seus usos (OLIVEIRA et al., 1982b; BRITO, 1993).

De acordo com Lepage (1986) e Lima et al. (2007), a madeira é um biopolímero tridimensional, constituída principalmente por celulose, hemicelulose e lignina (componentes primários), responsáveis pela formação da parede celular e pela maioria de suas propriedades, e pelos extrativos e cinzas (componentes complementares ou secundários). 
A análise química elementar da madeira revela que a mesma é constituída, principalmente, dos seguintes elementos químicos: oxigênio (44 \%), hidrogênio ( $9 \%$ ) e carbono (50\%), e considerando que os seus componentes primários são basicamente constituídos por esses elementos, o fenômeno da carbonização pode ser explicado e entendido a partir das transformações sofridas pelos três principais componentes da madeira (OLIVEIRA, 2003).

Segundo Oliveira et al. (1982), Almeida; Rezende (1992), apud Assis et al. (2008), a carbonização objetiva aumentar o teor de carbono fixo na madeira por meio de tratamento térmico, tornando-se necessário estudar a degradação sofrida pela celulose, hemiceluloses e lignina. A degradação da celulose se processa rapidamente em um curto intervalo de temperatura, cerca de $50{ }^{\circ} \mathrm{C}$, provocando drásticas mudanças no seu comportamento, com a perda de cerca de $77 \%$ do seu peso. As hemiceluloses começam a perder peso em temperaturas próximas a $225{ }^{\circ} \mathrm{C}$, sendo o componente menos estável da madeira, uma vez que sua degradação é quase completa na temperatura de $325{ }^{\circ} \mathrm{C}$, perdendo peso continuamente sob a ação do calor. A lignina é o componente da madeira mais difícil de se isolar, por apresentar uma estrutura relativamente complexa. Esta é o componente químico da madeira mais importante quando se pretende a produção de carvão vegetal, devido ao rendimento gravimétrico estar diretamente relacionado com o conteúdo de lignina na madeira. Em uma faixa de temperatura com variação entre 450 a $550{ }^{\circ} \mathrm{C}$, obtém um rendimento em carvão vegetal de $55 \%$ (SARKANEN; LUDWIG, 1971; OLIVEIRA et al., 1982 a).

As melhores propriedades químicas desejáveis do carvão, como maiores teores de carbono fixo e menores teores em substâncias voláteis e cinzas, estão associadas à madeira com altos teores de lignina, para determinadas condições de carbonização (temperatura final e taxa de aquecimento). Cada faixa de temperatura gera um produto diferente, sendo que a temperatura final tem grande influência na qualidade do carvão vegetal (Oliveira, 2003; Oliveira et al., 2010).

Em virtude da utilização diversa da madeira e da carência de informações sobre a vegetação da Caatinga, torna-se evidente a importância dos conhecimentos relativos às características da madeira e do carvão. Em razão dessa importância, essa pesquisa teve como objetivo fazer a caracterização físico-química e energética da madeira e do carvão vegetal de duas espécies de ocorrência no semiárido brasileiro, a fim de criar um banco de dados das espécies presentes no Bioma Caatinga e, consequentemente, indicar as espécies com potencial energético e posteriormente diminuir os impactos negativos causados sobre o ecossistema Caatinga.

\section{MATERIAL E MÉTODOS}

\section{Espécies selecionadas, coleta e amostragem das madeiras}

Foram avaliadas madeiras de Poincianella pyramidalis Tul. L. P. Queiroz e Handroanthus impertiginosus (Mart. ex DC.) Mattos, sendo selecionadas cinco árvores de cada espécie, que apresentavam as melhores características fisiológicas. A madeira foi coletada na Fazenda Santa Bárbara $\left(06^{\circ} 54^{\prime} 55^{\prime \prime} \mathrm{S}\right.$; $37^{\circ} 05^{\prime} 55^{\prime \prime} \mathrm{W}$ a 265 $\mathrm{m}$ de altitude), localizada no município de São Mamede-PB.

De cada árvore, foram retirados toretes com $30 \mathrm{~cm}$ de altura a 0 (base), 25, 50, 75 e $100 \%$ da altura comercial do tronco, considerada até $5 \mathrm{~cm}$ de diâmetro, sendo medidos os diâmetros nessas posições, para o cálculo do volume de madeira. Em seguida, foram retirados discos de $2,5 \mathrm{~cm}$ na porção mediana de cada torete. Essas amostras foram devidamente identificadas e transportadas para o Setor de Tecnologia de Produtos Florestais (STPF) da Unidade Acadêmica de Engenharia Florestal (UAEF), Universidade Federal de Campina Grande (UFCG), Campus de Patos-PB. Da porção mediana de cada torete, foi retirado um disco de $2,5 \mathrm{~cm}$ de espessura, subdividido em quatro partes, em forma de cunha, passando pela medula. Duas cunhas opostas foram utilizadas para a determinação da densidade básica e as restantes foram reservadas para estudos químicos e energéticos.

\section{Determinações das características físicas e químicas das madeiras selecionadas}

A densidade básica foi determinada de acordo com o método da balança hidrostática, descrito por Vital (1984). Para a determinação da densidade básica média de cada árvore utilizouse a média ponderada, tomando-se o volume entre as seções de onde foram retirados os discos como fator de ponderação. Após a secagem ao ar, as amostras destinadas às análises químicas foram transformadas em serragem em moinho tipo Wiley. A análise química da madeira foi efetuada em 
duplicata, na fração de serragem, classificadas em peneiras de 40/60 mesh, conforme as seguintes normas: preparação da madeira para análises químicas, conforme as normas TAPPI T 257 om - 85 (TAPPI, 1996) e ASTM, 1994; determinação do teor absolutamente seco das amostras de acordo com a norma TAPPI T 264 om - 88 (TAPPI, 1996); teor de extrativos totais, conforme a norma TAPPI T $264 \mathrm{om}$ - 88 (TAPPI, 1996), com a substituição do benzeno por tolueno; o teor de lignina foi determinado pelo método Klason, modificado de acordo com o procedimento proposto por Gomide e Demuner (1986). Do filtrado restante da análise da lignina Klason, foi feita a leitura em espectrofotômetro, para determinação da lignina solúvel em ácido, conforme Goldschimid (1971). O teor de lignina total é a soma da lignina residual mais a lignina solúvel em ácido. $\mathrm{O}$ teor de holocelulose foi obtido por diferença [\% holocelulose $=100-$ (teor de extrativo + teor de lignina + cinzas na madeira)].

A determinação do teor de cinzas ou minerais da madeira foi efetuada segundo a norma ABTCP M-11/77 (ABTCP, 1974). O poder calorífico superior foi determinado por meio de calorímetro adiabático de acordo com a norma ABNT NBR 8633 (ABNT, 1983).

\section{Carbonização da madeira e análise do carvão vegetal}

As carbonizações foram realizadas em um forno elétrico (mufla) adaptado para essa operação, com controle de temperatura. O controle de aquecimento foi manual, seguindo a marcha $\left(150{ }^{\circ} \mathrm{C}-1 \mathrm{~h} ; 200{ }^{\circ} \mathrm{C}-1 \mathrm{~h} ; 250{ }^{\circ} \mathrm{C}-1,5 \mathrm{~h}\right.$; $350{ }^{\circ} \mathrm{C}-1,5 \mathrm{~h}$; e $\left.450{ }^{\circ} \mathrm{C}-0,5 \mathrm{~h}\right)$. Foram carbonizados $320 \mathrm{~g}$, aproximadamente, de cavacos anidro, com duas repetições por árvore, totalizando dez carbonizações por espécie. Os vapores/gases foram conduzidos para um condensador tubular, com recolhimento do líquido pirolenhoso.

Após a carbonização, o rendimento gravimétrico, em carvão e em condensados, foi determinado, ao dividir a massa do carvão, seco ou do condensado, produzido pela massa da madeira seca.

As análises do carvão foram realizadas em duplicatas, sendo feitas análises químicas imediatas, determinação do rendimento em carbono fixo, determinação da densidade verdadeira e densidade aparente. A composição química imediata foi realizada conforme ABNT NBR 8112 (ABNT, 1983), com determinações de matérias voláteis, teor de cinzas e teor de carbono fixo, em base seca. O rendimento em carbono fixo foi obtido pelo produto entre teor de carbono fixo e rendimento gravimétrico da carbonização. As densidades verdadeira e aparente do carvão foram calculadas de acordo com as ASTM-D-167-73, adaptadas por Oliveira et al. (1982b). O poder calorífico superior foi determinado por meio de um calorímetro adiabático, conforme a Norma ABNT NBR 8633 (ABNT, 1983).

$\mathrm{Na}$ avaliação do experimento, utilizou-se o delineamento inteiramente casualizado, com dois tratamentos (espécies) e cinco repetições (árvores), com duas e quatro replicações por indivíduo para as análises da madeira e do carvão, respectivamente.

\section{RESULTADOS E DISCUSSÃO}

\section{Caracterizações físico-químicas das espécies Poincianella pyramidalis e Handroanthus impertiginosus}

A espécie Poincianella pyramidalis apresentou densidade básica média (DBM) de $\left(1.052,46 \mathrm{~kg} . \mathrm{m}^{-3}\right)$ superior à espécie Handroanthus impertiginosus $\left(997,75 \mathrm{~kg} \cdot \mathrm{m}^{-3}\right)$. Os valores da DBM encontrados para as espécies estudadas são superiores aos apresentados por Oliveira (2003), ao estudar as seguintes espécies de ocorrência na Caatinga: Mimosa tenuiflora $\left(929,47 \mathrm{~kg} \cdot \mathrm{m}^{-3}\right)$, Aspidosperma pyrifolium $\left(813,22 \mathrm{~kg} \cdot \mathrm{m}^{-3}\right)$ e Croton sonderianus $\left(683,81 \mathrm{~kg} \cdot \mathrm{m}^{-3}\right)$, usando metodologia semelhante. Almeida (2010) ao estudar as espécies Amburana cearensis e Piptadenia stipulacea, também presentes na Caatinga, encontrou valores médios de $1.001,93 \mathrm{~kg} \cdot \mathrm{m}^{-3}$ e $631,81 \mathrm{~kg} \cdot \mathrm{m}^{-3}$, respectivamente. Os valores da DBM apresentados para as espécies ensaiadas foram superiores aos exibidos por Vale et al. (2010), para as seguintes espécies lenhosas de Cerrado: Pterodon pubescens (820 kg.m $\left.{ }^{-3}\right)$, Dalbergia miscolobium (800 kg.m ${ }^{-3}$ ), Sclerolobium paniculatum $\left(780 \quad \mathrm{~kg} . \mathrm{m}^{-3}\right), \quad$ Stryphnodendron adstringens $\left(610 \mathrm{~kg} \cdot \mathrm{m}^{-3}\right)$ e Vochysia thyrsoidea $(580$ $\left.\mathrm{kg} \cdot \mathrm{m}^{-3}\right)$.

A espécie Poincianella pyramidalis apresentou o valor médio de cinzas $(3,69 \%)$ e extrativos totais $(11,81 \%)$, superiores aos da espécie Handroanthus impertiginosus $(0,87$ e $6,88 \%$, respectivamente) e valores médios inferiores em relação aos teores de lignina total $(24,64 \%)$ e poder calorífico superior da madeira $(4.413,50 \mathrm{kcal}$. $\mathrm{kg}^{-1}$ ), significativos estatisticamente em $1 \%$ de probabilidade. Não houve diferença estatística entre as espécies para o teor de holocelulose (Tabela 1). 
TABELA 1: Valores médios das características físico-químicas e poder calorífico superior da madeira de Poincianella pyramidalis e Handroanthus impertiginosus.

TABLE 1: Medium values of physicochemical characteristics and superior calorific power wood of Poincianella pyramidalis and Handroanthus impertiginosus.

\begin{tabular}{|c|c|c|c|c|c|c|c|}
\hline Espécie & Indivíduo & $\begin{array}{c}\mathrm{DBM} \\
\left(\mathrm{kg} \cdot \mathrm{m}^{-3}\right)\end{array}$ & $\begin{array}{c}\text { CIZM } \\
(\%)\end{array}$ & $\begin{array}{c}\text { EXT TOT } \\
(\%)\end{array}$ & $\begin{array}{l}\text { LIG TOT } \\
(\%)\end{array}$ & $\begin{array}{c}\mathrm{HOL} \\
(\%)\end{array}$ & $\begin{array}{c}\text { PCSM } \\
\left(\mathrm{kcal}^{\left.\mathrm{k} \mathrm{kg}^{-1}\right)}\right.\end{array}$ \\
\hline \multirow{5}{*}{ Poincianella pyramidalis } & 1 & 983,78 & 3,61 & 9,97 & 23,83 & 62,59 & 4560,50 \\
\hline & 2 & 1049,2 & 3,14 & 10,53 & 22,03 & 64,30 & 4490,00 \\
\hline & 3 & 1129,77 & 3,93 & 15,30 & 26,47 & 54,30 & 4434,50 \\
\hline & 4 & 1024,51 & 3,92 & 14,03 & 25,32 & 56,73 & 4194,50 \\
\hline & 5 & 1075,05 & 3,84 & 9,20 & 25,57 & 61,39 & 4388,00 \\
\hline \multicolumn{2}{|c|}{ Média } & $1052,46 a$ & $3,69 \mathrm{~A}$ & $11,81 \mathrm{~A}$ & $24,64 \mathrm{~B}$ & $59,86 a$ & $4413,50 \mathrm{~B}$ \\
\hline \multirow{5}{*}{$\begin{array}{l}\text { Handroanthus } \\
\text { impertiginosus }\end{array}$} & 1 & 963,77 & 0,73 & 6,42 & 25,55 & 67,30 & 4584,50 \\
\hline & 2 & 1010,02 & 0,66 & 6,55 & 28,53 & 64,26 & 4812,00 \\
\hline & 3 & 986,05 & 0,80 & 7,20 & 30,30 & 61,70 & 4920,50 \\
\hline & 4 & 985,51 & 1,01 & 6,70 & 28,82 & 63,47 & 4864,50 \\
\hline & 5 & 1043,39 & 1,13 & 7,55 & 28,78 & 62,54 & 4850,00 \\
\hline \multicolumn{2}{|c|}{ Média } & $997,75 a$ & $0,87 \mathrm{~B}$ & $6,88 \mathrm{~B}$ & $28,40 \mathrm{~A}$ & $63,85 \mathrm{a}$ & $4806,30 \mathrm{~A}$ \\
\hline \multicolumn{2}{|l|}{ QMRes } & 1958,85 & 0,07 & 3,73 & 3,03 & 11,10 & 18009,98 \\
\hline \multicolumn{2}{|l|}{ CV $(\%)$} & 4,32 & 12,00 & 20,66 & 6,56 & 5,39 & 2,91 \\
\hline
\end{tabular}

Em que: $\mathrm{DBM}=$ densidade básica média, $\mathrm{CIZM}=$ cinzas da madeira, $\mathrm{EXT}$ TOT $=$ Extrativos totais, $\mathrm{LIG}$ TOT $=$ lignina total, $\mathrm{HOL}=$ holocelulose, $\mathrm{PCSM}=$ poder calorífico superior da madeira, QMRes $=$ quadrado médio do resíduo e $\mathrm{CV}=$ coeficiente de variação. Médias na coluna seguidas de letra minúscula ou maiúscula igual não diferem estatisticamente $(\mathrm{p}<5$ e $1 \%$, respectivamente) pelo teste de Tukey.

O valor médio de cinzas da espécie Poincianella pyramidalis foi superior ao encontrado por Medeiros Neto (2008) para as espécies Anadenanthera colubrina (2,5 \%), Tabebuia aurea $(0,68 \%)$ e Amburana cearensis (2,05\%) e a valores encontrados por Oliveira (2003) para as espécies Croton sonderianus (0,68\%), Mimosa tenuiflora (0,56 \%), Aspidosperma pyrifolium $(0,56 \%)$ e Eucalyptus grandis $(0,45 \%)$, ambos usando metodologias semelhantes e espécies da Caatinga. Oliveira (2003) enfatiza que um maior percentual de cinzas na madeira está associado a um menor teor de lignina, fato esse observado com as espécies estudadas.

O teor de extrativos totais médio da espécie Poincianella pyramidalis (11,81\%) é superior ao encontrado por Santos (2008), ao analisar as madeiras de cinco espécies de ocorrência no Cerrado, cujos resultados variaram de 6 a $8 \%$.

A espécie Handroanthus impertiginosus apresentou o maior poder calorífico da madeira, com valor médio de $4.806,30 \mathrm{kcal} . \mathrm{kg}^{-1}$. Isso pode ser explicado, segundo Browing (1963), apud Cunha et al. (1989), ao afirmar que o poder calorífico é maior quando se verifica o aumento nos teores de lignina e extrativos, porque esses constituintes químicos da madeira contêm menos oxigênio que os polissacarídeos presentes na holocelulose.

A espécie Poincianella pyramidalis apresentou o maior teor de rendimento gravimétrico $(43,03 \%)$. No entanto, a Handroanthus impertiginosus exibiu um maior teor de carbono fixo $(67,68 \%)$, fato explicado por esta espécie apresentar os menores teores de matérias voláteis $(30,36 \%)$ e cinzas $(1,96 \%)$ (Tabela 2$)$.

Oliveira et al. (2010) demonstraram que o teor de carbono fixo é inversamente proporcional ao teor de matérias voláteis. $\mathrm{O}$ rendimento gravimétrico das espécies estudadas foi superior aos valores encontrados por Vale et al. (2010) para cinco espécies de ocorrência no Cerrado, os quais variaram de 25 a $28 \%$. A espécie Poincianella pyramidalis apresentou um rendimento em carvão médio (43,03\%) superior aos valores encontrados por Vale; Nogueira (2001), ao estudarem 12 
espécies de ocorrência no Bioma Cerrado, cujos resultados variaram de 32 a $39 \%$ e aos valores encontrados por Oliveira (2003) para as espécies Croton sonderianus (35,68 \%), Mimosa tenuiflora $(39,70 \%)$ e Aspidosperma pyrifolium $(34,96 \%)$ e a valores encontrados por Almeida (2010) ao avaliar a madeira de Amburana cearensis (38,39\%) e Piptadenia stipulacea (38,34\%), ambos trabalharam com espécies de ocorrência na Caatinga.

Segundo Petroff; Doat (1978), citados por Oliveira et al. (2010), maiores teores de lignina e extrativos na madeira ocasionam um maior rendimento em carvão vegetal, em virtude de serem estes constituintes químicos ricos em carbono. Apesar de a espécie Poincianella pyramidalis apresentar uma menor quantidade de lignina, o seu conteúdo de extrativos é superior ao encontrado para a espécie Handroanthus impertiginosus.

Nota-se que a densidade aparente do carvão vegetal foi superior para a espécie de maior densidade básica da madeira, fato observado em relação à madeira de Poincianella pyramidalis $\left(0,60\right.$ g.cm $\left.{ }^{-3}\right)$. Silva et al. (2007) e Sturion et al. (1988) constataram a mesma observação ao estudarem espécies da Amazônia e Eucaliptos, respectivamente.

Observa-se, também, que houve uma diminuição no teor de carbono fixo e um aumento no teor de matérias voláteis, com o aumento do rendimento em carvão para a espécie Poincianella pyramidalis. Vale; Nogueira (2001), ao analisarem a qualidade do carvão vegetal de 12 espécies de ocorrência no Cerrado, constataram a mesma observação.

A espécie Poincianella pyramidalis exibiu um teor de cinzas do carvão $(5,27 \%)$ elevado. Oliveira et al. (2006) encontraram um valor de cinzas do carvão (1,32 \%) para a espécie Mimosa tenuiflora. Essa característica é importante, pois serão necessárias mais limpezas em fornalhas e em outros sistemas onde a madeira é utilizada como fonte de energia, provocando danificação dos equipamentos.

TABELA 2: Valores médios das análises do carvão vegetal das espécies Poincianella pyramidalis e Handroanthus impertiginosus.

TABLE 2: Medium values of charcoal analyses of species Poincianella pyramidalis and Handroanthus impertiginosus.

\begin{tabular}{ccccccccccc}
\hline Espécie & Ind. & $\begin{array}{c}\text { RG } \\
(\%)\end{array}$ & $\begin{array}{c}\text { RLC } \\
(\%)\end{array}$ & $\begin{array}{c}\mathrm{DA} \\
\left(\mathrm{g} / \mathrm{cm}^{3}\right)\end{array}$ & $\begin{array}{c}\mathrm{DV} \\
\left(\mathrm{g} / \mathrm{cm}^{3}\right)\end{array}$ & $\begin{array}{c}\mathrm{MV} \\
(\%)\end{array}$ & $\begin{array}{c}\text { CIZC } \\
(\%)\end{array}$ & $\begin{array}{c}\mathrm{CF} \\
(\%)\end{array}$ & $\begin{array}{c}\text { RCF } \\
(\%)\end{array}$ & $\begin{array}{c}\text { PCSC } \\
(\mathrm{kcal} / \mathrm{kg})\end{array}$ \\
\hline & 1 & 42,01 & 37,93 & 0,513 & 1,272 & 34,508 & 5,472 & 60,020 & 25,21 & 6450,00 \\
Poincianella & 2 & 42,58 & 41,28 & 0,652 & 1,264 & 34,390 & 5,594 & 60,015 & 25,55 & 6135,00 \\
pyramidalis & 3 & 44,14 & 37,20 & 0,610 & 1,253 & 34,108 & 5,297 & 60,595 & 26,75 & 5937,00 \\
& 4 & 44,55 & 37,66 & 0,663 & 1,244 & 33,925 & 5,775 & 60,300 & 26,86 & 5997,00 \\
& 5 & 41,87 & 41,24 & 0,560 & 1,266 & 33,823 & 4,194 & 61,983 & 25,95 & 6220,00 \\
Média & & $43,03 \mathrm{~A}$ & $39,06 \mathrm{~b}$ & $0,60 \mathrm{a}$ & $1,26 \mathrm{a}$ & $34,150 \mathrm{~A}$ & $5,27 \mathrm{~A}$ & $60,582 \mathrm{~B}$ & $26,04 \mathrm{a}$ & $6247,80 \mathrm{~B}$ \\
\hline & 1 & 37,61 & 41,78 & 0,455 & 1,172 & 29,679 & 2,122 & 68,199 & 25,65 & 6970,00 \\
Handroanthus & 2 & 37,26 & 43,78 & 0,546 & 1,108 & 30,850 & 1,600 & 67,550 & 25,17 & 7052,00 \\
impertiginosus & 3 & 37,41 & 43,02 & 0,492 & 1,166 & 29,196 & 1,823 & 68,981 & 25,81 & 6970,00 \\
& 4 & 38,36 & 41,99 & 0,519 & 1,264 & 31,378 & 1,947 & 66,675 & 25,58 & 6946,00 \\
& 5 & 38,86 & 41,27 & 0,468 & 1,270 & 30,714 & 2,295 & 66,991 & 26,03 & 6949,00 \\
Média & $37,90 \mathrm{~B}$ & $42,37 \mathrm{a}$ & $0,50 \mathrm{~b}$ & $1,20 \mathrm{a}$ & $30,360 \mathrm{~B}$ & $1,96 \mathrm{~B}$ & $67,679 \mathrm{~A}$ & $25,65 \mathrm{a}$ & $6977,40 \mathrm{~A}$ \\
\hline QMRes & & 1,00 & 2,56 & 0,003 & 0,002 & 0,45 & 0,231 & 0,77 & 0,315 & 21480,25 \\
\hline CV(\%) & & 2,47 & 3,93 & 9,45 & 4,05 & 2,07 & 13,30 & 1,37 & 2,17 & 2,23 \\
\hline
\end{tabular}

Em que: Ind = Indivíduo, $\mathrm{RG}=$ rendimento gravimétrico, $\mathrm{RLC}=$ rendimento em líquido condensado, $\mathrm{DA}=\mathrm{densidade}$ aparente, $\mathrm{DV}=$ densidade verdadeira, $\mathrm{MV}=$ matérias voláteis, $\mathrm{CIZ}=$ cinzas do carvão, $\mathrm{CF}=\mathrm{carbono}$ fixo, $\mathrm{RCF}=$ rendimento em carbono fixo e PCSC $=$ poder calorífico superior do carvão, QMRes = quadrado médio do resíduo, $\mathrm{CV}=$ coeficiente de variação. Médias na coluna seguidas de letra minúscula ou maiúscula igual não diferem estatisticamente $(\mathrm{p}<5$ e $1 \%$, respectivamente) pelo teste de Tukey. 
O maior teor de carbono fixo foi encontrado para a espécie Handroanthus impertiginosus $(67,68 \%)$. No entanto, apresentou um menor rendimento em carbono fixo do carvão $(25,65 \%)$, estatisticamente semelhante à espécie Poincianella pyramidalis. Isso pode ser explicado, segundo Brito; Barrichelo (1977), pelo fato de que os maiores teores de carbono fixo, nos carvões produzidos a partir das madeiras mais lignificadas, são decorrência do fato de a lignina possuir cerca de $65 \%$ de carbono elementar (C) em sua composição contra $45 \%$ de $\mathrm{C}$ que ocorre normalmente nos polissacarídeos, celulose e hemicelulose.

Oliveira (2003), ao estudar as espécies Croton sonderianus, Mimosa tenuiflora, Aspidosperma pyrifolium e Eucalyptus grandis, encontrou valores de carbono fixo que variaram de 70 a $72 \%$, superiores aos exibidos para as espécies Poincianella pyramidalis e Handroanthus impertiginosus.

A espécie Handroanthus impertiginosus apresentou, em média, um maior poder calorífico do carvão $\left(6.977,40 \mathrm{kcal} \cdot \mathrm{kg}^{-1}\right)$. Esse valor superou o encontrado por Oliveira et al. (2006), ao estudarem a espécie Mimosa tenuiflora, com valor médio de $6.866 \mathrm{kcal} . \mathrm{kg}^{-1}$, madeira considerada de bom potencial para a produção de carvão e geração de energia.

\section{CONCLUSÕES}

A densidade básica da espécie Poincianella pyramidalis foi superior a da Handroanthus impertiginosus, porém, ambas são estatisticamente semelhantes.

A Poincianella pyramidalis apresentou maiores teores de cinzas da madeira extrativos totais, menores teores de lignina e poder calorífico superior da madeira que a Handroanthus impertiginosus, exceto para os teores de holocelulose, que foram semelhantes.

O carvão vegetal produzido com a madeira da Poincianella pyramidalis apresentou maiores teores de rendimento gravimétrico, densidade aparente e cinzas do carvão e menores teores de carbono fixo e matérias voláteis, quando comparada à espécie Handroanthus impertiginosus. O rendimento em carbono fixo e a densidade verdadeira do carvão foram semelhantes.

O elevado teor de cinzas do carvão da espécie Poincianella pyramidalis influenciou a um menor teor de carbono fixo. No entanto, o rendimento em carbono fixo foi superior, devido à relação direta com o rendimento gravimétrico.

Pode-se concluir que as duas espécies de ocorrência no semiárido Nordestino, apresentam boas características para produção de carvão. No entanto, de modo geral, em razão das características físicas, químicas e energéticas da madeira, o carvão vegetal obtido da espécie Handroanthus impertiginosus apresentou as melhores propriedades, visando a fins energéticos.

\section{AGRADECIMENTOS}

A Coordenação de Aperfeiçoamento de Pessoal de Nível Superior (CAPES) e ao Conselho Nacional de Desenvolvimento Científico e Tecnológico (CNPq) pela concessão do apoio financeiro ao primeiro e quarto autor, respectivamente.

\section{REFERÊNCIAS BIBLIOGRÁFICAS}

ABCTP-Associação Brasileira Técnica De Celulose e Papel. NORMAS ABTCP. Normas Técnicas ABCTP. São Paulo, ABTCP, 1974. n.p.

ABNT - Associação Brasileira de Normas Técnicas. Normas Técnicas NBR 8112. Brasília, 1983. n.p. ABNT - Associação Brasileira de Normas Técnicas. Normas Técnicas NBR 8633. Brasília, 1983. n.p. ALMEIDA, A. M. C. Avaliação anatômica, físicoquímica e energética da madeira das espécies Piptadenia stipulacea (Benth.) Ducke e Amburana cearensis(Allemao) A. C. Smith de ocorrência no semiárido nordestino brasileiro. 2010. $40 \mathrm{f}$. Dissertação (Mestrado em Ciências Florestais)Universidade Federal de Campina Grande, Paraíba, 2010.

AMERICAN SOCIETY FOR TESTING AND MATERIALS. ASTM. Wood. Annual Book of ASTM Standards, Philadelphia, v. 410, 1994.

ANDRADE, A. S. Qualidade da madeira, celulose e papel em Pinus taeda l: influência da idade e classe de produtividade. 2006. 94 f. Dissertação (Mestrado em Ciências Florestais)-Universidade Federal do Paraná, Curitiba, 2006.

ANDRADE, L. A. et al. Análise da cobertura de duas fitofisionomias de Caatinga, com diferentes históricos de uso, no município de São João do Cariri, Estado da Paraíba. Cerne, Lavras, v. 11, n. 3, p. 253-262, jul./set. 2005.

ARAÚJO, L. V. C. et al. Características dendrométricas e densidade básica da jurema-preta 
(Mimosa tenuiflora (Willd.) Poir.) de duas regiões do estado da Paraíba. Revista Caatinga, Mossoró, v. 20, n. 1, p. 89-96, jan./mar.2007.

ASSIS, C. O.et al. Sistema alternativo para carbonização de madeira. Scientia Forestalis, Piracicaba, v. 36, n. 78, p. 133-140, jun. 2008.

BRITO, J. O. Reflexões sobre a qualidade do carvão vegetal para uso siderúrgico. IPEF, Piracicaba, 1993. 6 p. (Circular técnica, 181).

BRITO, J. O.; BARRICHELO, L. E. G.. Correlação entre as características físicas e químicas da madeira e a produção de carvão: 1 densidade e teor de lignina na madeira de eucalipto. IPEF, Piracicaba, n. 14, p. 9-20, 1977.

BURGER, L. L.; RICHTER, H. G.Anatomia da Madeira. São Paulo: Nobel, 1991. 154 p.

CUNHA, M. P. S. C. et al. Estudo químico de 55 espécies lenhosas para geração de energia em caldeiras. In: ENCONTRO BRASILEIRO EM MADEIRAS E EM ESTRUTURAS DE MADEIRA, 3., 1989, São Carlos. Anais... São Carlos: 1989. v. 2, p. 93-121.

GOLDSCHIMID, O. Ultraviolet spectra. In: SARKANEN, K. V.; LUDWWIG, C. H. (Eds) Lignins. New York, Wiley - Interscience, 1971. p. 241-66.

GOMIDE, J. L.; DEMUNER, B. J. Determinação do teor de lignina em material lenhoso: método Klason modificado. O PAPEL, São Paulo, v. 47, n. 8, p. 36-38, 1986.

JOHNSON, D. V. Relatório sobre o manejo da caatinga, semi-árido do Nordeste Brasileiro. In: PROJETO PNUD/FAO/BRA/85/007. Natal: 1985. (Circular Técnica, 3).

LEPAGE. E.S. Química da Madeira. In: LEPAGE, E. S. (Coord.). Manual de preservação de madeira. São Paulo: IPT, 1986. v. 1, p. 69-97.

LIMA, J. T. et al. Variation in wood density and mechanical properties in Eucalyptus clones. In: THE FUTURE OF EUCALYPTS FOR WOOD PRODUCTS. 2000, Launceston. Procceedings... Launceston: IUFRO., 2000, p. 282-291.

LIMA, S. R. et al. Estudo dos constituintes macromoleculares, extrativos voláteis e compostos fenólicos da madeira de candeia - Moquinia polymorpha (Less.) DC. Ciência Florestal, Santa Maria, v. 17, n. 2, p. 145-155, abr./jun.. 2007.

LORENZI, H. Árvores brasileiras: manual de identificação e cultivo de plantas arbóreas nativas do Brasil, 4. ed., v. 1, Nova Odessa: Instituto Plantarum, 2002. $368 \mathrm{p}$.

MAIA, G. N. Caatinga: árvores e arbustos e suas utilidades. São Paulo: D\&Z Computação, 2004. $413 \mathrm{p}$.

MEDEIROS NETO, P. N. Correlação entre teores de extrativos e a resistência natural de quatro madeiras a cupins xilófagos. 2008, 27 f.Trabalho de conclusão de curso (Graduação em Engenharia Florestal)-Universidade federal de Campina Grande, Paraíba, 2008.

MELO, R. R. et al.Estudo da variação radial da densidade básica de sete madeiras do Semi-Árido. Revista Científica Eletrônica de Engenharia Florestal, Garça, n. 7, fev. 2006.

MMA- Ministério do Meio Ambiente. Mapa de cobertura vegetal dos Biomas brasileiros. Brasília, DF, 2007. 17 p.

OLIVEIRA, A. C. et al. Parâmetros de qualidade da madeira e do carvão vegetal de Eucalyptus pellita $\mathrm{F}$. Muell. Scientia Forestalis, Piracicaba, v. 38, n. 87, p. 431-439, set. 2010.

OLIVEIRA, E. Características anatômicas, químicas e térmicas da madeira de três espécies de maior ocorrência no semiárido nordestino. 2003. 122 p. Tese (Doutorado em Ciências Florestais)-Universidade Federal de Viçosa, Viçosa, 2003.

OLIVEIRA, E.et al. Estrutura anatômica da madeira e qualidade do carvão de Mimosa tenuiflora (Willd.) Poir. Revista Árvore, Viçosa, v. 30, n. 2, p. 311318, 2006.

OLIVEIRA, J. B. et al. Produção de carvão vegetal - aspectos técnicos. In: PENEDO, W. R. (Ed.) Produção e utilização de carvão vegetal. Belo Horizonte: CETEC. 1982a. p. 60-73.

OLIVEIRA, J. B. et al. Propriedades do carvão vegetal. In: PENEDO,W. R. (Ed.) Carvão vegetal: destilação, carvoejamento, controle de qualidade. Belo Horizonte: CETEC. 1982b. p.39-61.

OLIVEIRA, J. T. et al. Tecnologias aplicadas ao setor madereiro II. Jerônimo Monteiro : Ed. Aquarius,2007. $302 \mathrm{p}$.

PAULA, J. E.; ALVES, J. L. H. 897 Madeiras nativas do Brasil: anatomia - dendrologia dendrometria - produção - uso. Porto Alegre, Cinco Continentes, 2007. $438 \mathrm{p}$.

PINHEIRO, P. C. C.; SÉYE, O. Influência da temperatura de carbonização nas propriedades do carvão vegetal de Eucalyptus. In: CONGRESSO ANUAL DA ABM, 53.,1998, Belo Horizonte. Disponível em:< (http://www.demec.ufmg.br/ professor/paulocpinheiro/papers/abm98.pdf. ) > Acessoem: 15 de fevereiro de 2010.

SABONARO, D. Z.; GALBIATTI, J. A. Efeito de 
níveis de irrigação em substratos para a produção de mudas de ipê-roxo. Scientia Forestalis, n. 74, p. 95-102, 2007.

SANTOS, I. D. Influência dos teores de lignina, holocelulose e extrativos na densidade básica, contração da madeira e nos rendimentos e densidade do carvão vegetal de cinco espécies lenhosas do cerrado. 2008. 57 f. Dissertação (Mestrado em Ciências Florestais)-Universidade de Brasília, Brasília, 2008.

SARKANEN, K. V.; LUDWIG, C. H. Lignins: occurrence, formation, structure and reation. New York: John Wiley \& Sons, 1971. 916 p.

SILVA, M. G. et al. Carvão de resíduos de indústria madeireira de três espécies florestais exploradas no município de Paragominas, PA. Acta amazônica, v. 37. p. 61-70, 2007.

SIQUEIRA FILHO, J. A. et al. Guia de Campo de Árvores da Caatinga. Petrolina, 2009. 64 p.

SJÖSTRÖM, E. Wood chemistry: fundamentals and applications. $2^{\text {nd }}$ ed. New York: Academic Press. 1993. $293 \mathrm{p}$.

STURION, J. A. et al. Qualidade da madeira de Eucalyptus vimanalis para fins energéticos em função do espaçamento e idade de corte. Boletim de Pesquisa Florestal, Colombo, n. 16, p.55-59, 1988. SUDEMA - Superintendência de Administração do Meio Ambiente. Atualização do diagnóstico florestal do Estado da Paraíba. João Pessoa: SUDEMA, 2004. 268 p.

TAPPI - Technical Association of the Pulp and Paper Industry. TAPPI test methods T $257 \mathbf{c m}-85$ : sampling and preparing wood for analysis. Atlanta: Tappi Technology Park, 1996. v. 1

TAPPI - Technical Association of the Pulp and Paper Industry. TAPPI test methods T 264 om - 88: preparation of wood for chemical analysis. Atlanta: Tappi Technology Park, 1996. v. 1.

VALE, A. T. et al. Relações entre propriedades químicas, físicas e energéticas da madeira de cinco espécies de Cerrado. Ciência Florestal, Santa Maria, v. 20, n. 1, p. 137-145, jan./mar. 2010.

VALE, A. T.; NOGUEIRA. M. Carbonização de madeiras do Cerrado e análise do carvão vegetal. Revista Arvore, Viçosa, v. 25, n. 2, p. 271-276, 2001.

VALERIO, A. F. et al. Determinação da densidade básica da madeira de peroba (Aspidosperma polineuron Muell. Arg.) ao longo do fuste. Revista Caatinga, Mossoró, v. 21, n. 3, p. 54-58, jul./set. 2008.

VITAL, B. R. Métodos de determinação da densidade da madeira. Viçosa: SIF, 1984. 21 p. (Boletim técnico, 1). 